\title{
Germanica
}

\section{Bewältigungsinstrument Anti-Heimatliteratur}

"Anti-Heimatliteratur » et travail de mémoire

Ingeborg Rabenstein-Michel

\section{CpenEdition}

Journals

Édition électronique

URL : http://journals.openedition.org/germanica/525

DOI : 10.4000/germanica.525

ISSN : 2107-0784

Éditeur

Université de Lille

Édition imprimée

Date de publication : 1 juin 2008

Pagination : 157-169

ISBN : 978-2-913857-21-6

ISSN : 0984-2632

Référence électronique

Ingeborg Rabenstein-Michel, « Bewältigungsinstrument Anti-Heimatliteratur », Germanica [Online], 42 |

2008, Online erschienen am: 01 Juni 2010, abgerufen am 06 Oktober 2020. URL : http://

journals.openedition.org/germanica/525; DOI : https://doi.org/10.4000/germanica.525

Ce document a été généré automatiquement le 6 octobre 2020.

(c) Tous droits réservés 


\title{
Bewältigungsinstrument Anti- Heimatliteratur
}

\author{
«Anti-Heimatliteratur » et travail de mémoire
}

Ingeborg Rabenstein-Michel

Vielleicht war es gut, dass wir den Krieg verloren haben, sonst wären wir jetzt vielleicht Nazis ${ }^{1}$.

1 Literarische Quertreiberei hat in Österreich Tradition und Methode. Die ansehnliche Liste der Nestbeschmutzer, die für manche bereits mit den Predigten eines Abraham a Sancta Clara beginnt, reicht über Nestroy, Kraus und Horvath bis Turrini, Bernhard und Jelinek - um nur einige der bekanntesten Namen zu nennen - und ist voraussichtlich noch lange nicht abgeschlossen. Allen wurde und wird vorgeworfen, eine von der Institution als völlig ungerechtfertigt zurückgewiesene Kritik sowohl inhaltlich als auch formal auf allzu radikale Weise umzusetzen. Denn vor allem ab dem 20. Jahrhundert geht in dieser Literatur bzw. diesem Theater die gesellschaftliche Hinterfragung immer wieder Hand in Hand mit dem Aufbrechen der gängigen Gattungen, die systematisch dekonstruiert und um- bzw. neugestaltet werden ${ }^{2}$. Ziel: Die polemische Demaskierung der Brüche des Systems und der Fehlleistungen der Geschichte im Duktus einer akribisch-aggressiven Sprachkritik. Die „Öffentlichkeit“ reagiert im allgemeinen mit Verstörung und Abwehr auf die „Verunglimpfung“ der heiligsten Werte und Verdienste des Staates ${ }^{3}$, auf den „respektlosen“ Umgang mit dem Kunstkanon und den Ikonen des Bildungsbürgertums. Das Hauptanliegen dieser Literatur deckt sich also mit dem Hauptvorwurf, der ihr immer wieder gemacht wird: Die Weigerung, unkritisch apologetisch ein als fragwürdig empfundenes Gesellschaftsprinzip zu unterstützen, Verantwortung bzw. Rechenschaft zu fordern, und, seit dem Ende des Zweiten Weltkriegs, vor allem die konstante und für viele so besonders enervierende Infragestellung eines der Hauptpfeiler der Zweiten Republik Österreich, des Heimatbegriffs. So entwickelt sich nach 1945 „eine eigenständige, österreichische, in anderen Ländern nicht existierende Gattung ${ }^{4 \text {, }}$, die AntiHeimatliteratur. Dieser gewiss zu weit gefasste, nach Differenzierungen verlangende Sammelbegriff weist trotz allem auf den gemeinsamen Nenner äußerst verschiedener 
Werke hin: die kritische Analyse des als „Identität" neu erfassten Heimatbegriffs, ein Ansatz, der sich bis heute erhalten und sogar verschärft hat. Sicher nicht zu Unrecht wurde unterstrichen, dass für die letzten zwei Jahrzehnte trotz (oder vielleicht gerade wegen?) der starken Persönlichkeit der österreichischen Autoren keine determinierenden literarischen Strömungen zu verzeichnen sind und sich trotz ihrer außerordentlichen und grenzenüberschreitenden Vitalität eher das Bild einer „zersplitterten Literatur“ (littérature éclatée) ergibt ${ }^{5}$. Und doch kann Menasse auch 2005 noch schreiben, dass sich die Liste der Autoren, die diese Form herausgebildet und weiterentwickelt haben wie „ein beinahe vollständiges Who's who der modernen österreichischen Literatur ${ }^{6 “ " ~ l i e s t ~ u n d ~ i h r ~ z e i t g e n o ̈ s s i s c h e ~ W e r k e ~ v o n ~ S c h r i f t s t e l l e r n ~ w i e ~}$ Gstrein, Winkler, Jelinek oder Jonke zurechnen.

2 In der folgenden Untersuchung soll auf die Anti-Heimatliteratur der ersten Nachkriegsjahrzehnte eingegangen werden, die in formal heterogener Form thematisch auf zwei der fragwürdigsten österreichischen Nachkriegsstrategien reagiert: die Tabuisierung der störenden Vergangenheit und die global undistanzierte Übernahme eines nicht nur überholten, sondern historisch ausgiebigst missbrauchten Heimatbegriffs und dessen fragwürdige Neuinszenierung durch die Regierung des „neuen Österreich“ nach 1945 bzw. 1955 (Staatsvertrag / politische Souveränität) in Form des gerne herangezogenen Klischees der unschuldigen Natur. Diese „Heimat" entlastet stellvertretend die Menschen, die in ihr leben - „die Österreicher" - und befreit sie auf höchst gelegene Weise von der Aufgabe der Erinnerungsarbeit. Einige Überlegungen zum Begriff Heimat selbst sowie zum Thema Heimat und Erinnerung / Heimat als Museum versuchen in der Folge dazu beizutragen, die Funktion der AntiHeimatliteratur klarer zu umreißen.

\section{Wo die Erinnerung Bescheid weiß 7}

Das deutsche Wort Heimat umfasst bekanntlich einiges mehr als die rein topographischen Grenzen des Geburtslandes oder Geburtsortes. Es bezeichnet ein Umfeld, in dem das Zusammenspiel von Landschaften, Gegenständen, Gewohnheiten, Personen usw. weniger rationelle als vor allem emotionale Bezugspunkte erzeugt. Als Nicht-Ort bzw. als Utopie kann sie dementsprechend Berhard Schlink bezeichnen: „Denn die Erinnerung und die Sehnsüchte machen die Orte zur Heimat ${ }^{\text {"4 }}$. In der Erinnerung fühlt man sich daheim, wo man „natürlich“ Teil eines Ganzen war, im Gegensatz zur Verlorenheit in der immer beunruhigenden, potentiell unheimlichen und bedrohlichen Fremde. Das Gefühl selbst wird meistens als Ausdruck des Verlustes einer Vertrautheit bewusst. So wird Heimat zunehmend im 19. Jahrhundert thematisiert, einer Epoche also, wo sich als globale Antwort auf die zunehmende Entwurzelung und Entfremdung des Menschen im Kontext der fortschreitenden Industrialisierung und Verstädterung das Konzept der Heimatliteratur herausbildet. Die Komplexität des Lebens- und Arbeitsrhythmus in den Metropolen erzeugt Sehnsucht nach der Klarheit der warmen, schützenden Idylle, die man in der idealisierten Dorfgemeinde $z u$ finden vermeint. Der sinnzerstörenden Hektik der Großstadt wird so als Gegenpol ein sinnstiftendes „einfaches Leben“ entgegengestellt, die naturverbundene Verrichtung zeitloser Arbeitsprozesse, die sich so grundsätzlich von der anonymen Produktionsdynamik des Kapitalismus zu unterscheiden scheinen. Der Glaube an ein (notwendigerweise) moralisches Handeln in einer als intakt erklärten 
Natur wird in diesem Konstrukt als Postulat ebenso konstitutiv wie das unbedingte Vertrauen in die Unantastbarkeit der dort angeblich noch geltenden überlieferten Werte: erbaulich und verklärend tröstet die Heimatliteratur den verstörten Großstadtmenschen und gibt ihm den Glauben an das ewig Überdauernde zurück.

Ihre weitgehende thematische Übereinstimmung mit den völkischen Idealen ${ }^{9}$ des Dritten Reiches führte dazu, dass die Heimatliteratur von den Nationalsozialisten nicht nur übernommen, sondern auch kaum verändert propagandistisch eingesetzt werden konnte: In der Polarisierung Land - Großstadt sind Landschaften, Figuren und Werte der Heimatliteratur unbedingt und grundsätzlich deutsch, im absoluten Kontrast zu den kosmopolitischen Strömungen der „intellektuellen jüdischen Dekadenz“ der internationalen Metropolen. In diesem Kontext versteht sich dementsprechend auch eine der zahllosen Schikanen und Verordnungen, die den Juden von den Nazis auferlegt wurde: Das Verbot, $\operatorname{Tracht}^{10} \mathrm{zu}$ tragen (z.B. Salzburg, 1938). Als Symbol für Ursprünglichkeit, Tradition und Heimatverbundenheit signalisierte diese spezifische Kleidung Zugehörigkeit zur (deutschen) Volksgemeinde für die einen, und Ausschluss für die anderen - eine Aussagekraft, die der Tracht übrigens auch nach 1945 teilweise erhalten blieb. In der Anti-Heimatkunst der Nachkriegsjahrzehnte wurde den Autoren vor allem für die Bühnenwerke der Rückgriff auf die Tracht als unerträgliche visuelle Hervorhebung des kritischen Inhalts besonders angekreidet.

Das von der Heimatliteratur vor allem für den süddeutschen Raum (Österreich, Bayern) entworfene und im Dritten Reich demagogisch eingesetzte Bild einer irreell heilen Welt ist 1945 bzw. 1955 nicht nur anachronistisch, da es als solches nie existiert hatte, sondern auch, wie man hätte annehmen können, doppelt diskreditiert. Einerseits als nostalgisch-sentimentale Verklärung einer konstruierten Irrealität, andererseits durch den Missbrauch durch das nationalsozialistische Regime. Und doch wird schon im befreiten, jedoch noch besetzten Österreich, das seine Zugehörigkeit zum zukünftigen Westblock auf der Basis der von den Alliierten 1943 (Moskauer Deklaration) gewährten Opfertheorie zu erhandeln sucht, der Begriff Heimat als Instrument zur Erzeugung eines nationalen Zugehörigkeitsgefühls kaum neu belegt übernommen. Die institutionelle, streng auf die Zukunft ausgerichtete Wiederaufbaudynamik blendete so die Periode 1938-1945 zwar bewusst aus, bediente sich jedoch demonstrativ eines kaum modifizierten, fragwürdigen Heimatbegriffs als Anknüpfungs- und Abgrenzungsstrategie: Anknüpfung an ein „wahres“ Österreich, das historisch undeutlich, aber auf jeden Fall irgendwo vor 1938 angesetzt wurde, Abgrenzung zu Deutschland (und seiner erwiesenen Schuld) in dem noch sehr fragilen neuen Österreich. Im Nachkriegsland der Berge, das die Frage nach der Verantwortung der Menschen nach Möglichkeit zu vermeiden sucht, wird die topographische Komponente aufdringlich in den Vordergrund gespielt. Nicht zufällig werden bis weit in die siebziger Jahre in Ausstellungen, die Titel wie „Unser Österreich ${ }^{11 “}$ tragen, hauptsächlich Landschaft und Natur, höchst selten jedoch Menschen dargestellt, um die (Mit)Schuldfrage nach Möglichkeit zu umgehen. Heimat wurde so zur überwältigenden, unschuldigen und identitätsstiftenden Naturkulisse stilisiert, die vorteilhaft die historischen Fehlentscheidungen der Menschen verbarg, das heißt, dazu beitrug, die nach 1945 einsetzende allgemeine Amnesie effizient zu unterstützen. So kann der ewige Mitläufer und „gute alte Zeit ${ }^{12 “}$ - Nostalgiker Karl (eine der großen Figuren der Nestbeschmutzerliteratur) in Steirerweste und Steirerhut auch nach seiner 
ersten und einzigen Reise durch seine neue Heimat andächtig feststellen: „Schaun S', is do eigentlich schön: Österreich ${ }^{13}$.“

6 Bei diesem „eigentlich“ setzt nun aber die Kritik der neuen Gattung an. Eine sterile Kulisse ohne Menschen kann nicht Heimat sein. Heimat wird in und durch Erinnerung hergestellt, nicht nur glücklich-verklärende, sondern auch schmerzliche und störende. Und diese fehlt nach 1945, da die Menschen, die sich hätten erinnern können, von den neuen „Heimatmachern ${ }^{14 \text { “ }}$ bewusst ausgeblendet wurden ${ }^{15}$. In einer als bloßes Dekorum definierten Heimat kann die Erinnerung jedoch nicht mehr Bescheid wissen: Dieser Bruch zwischen einem sich kritisch erinnernden und einem selbstgefällig vergessenden Österreich verschärfte sich in den sechziger Jahren ${ }^{16}$ und gab der Anti-Heimatkunst den Anlass, diese „Anti-Heimat par excellence ${ }^{17 “}$ mit ihren grell überzeichneten (Kunst)Figuren, in denen sich „das Volk“ erstaunlich mühelos wiedererkannte ${ }^{18}$, wieder zu „bevölkern“.

\section{2. "Gemma, und sag ma, es war nix!19"}

7 Keine Menschen - keine Erinnerung, oder zumindest keine unbequemen Erinnerungen. Das Nachkriegsösterreich leidet dank der höchst opportunen Gründungsmythen der zweiten Republik (und allen voran des Opfermythos) an einem institutionell gebilligten Gedächtnisschwund im Bereich Mittäterschaft und Verantwortung. Gefordert wird von offizieller Seite nicht der Blick zurück, sondern ausnahmslos der alles vergessende bzw. vergebende Blick in die Zukunft. Als Antwort darauf macht sich die AntiHeimatliteratur zur Aufgabe, die ausgeblendete Vergangenheit in der Kunst wieder aufleben zu lassen. So ist sie als ein Prozess der provokativen Wiederherstellung von Erinnerung zu verstehen: Aichinger, Bachmann, Handke, Jonke, Lebert, Qualtinger, Fritsch, Turrini, Kerschbaumer, Mitgutsch, Kratz, Unger, Mitterer, Scharang, Innerhofer, Schindel, Reichart, Jelinek usw. protestieren geschlossen, wenn auch auf formal höchst differenzierte Weise, gegen eine „Heimat wie Weihnachten ${ }^{20 ", ~ g e g e n ~ d i e ~}$ öffentliche Inszenierung der Amnesie.

8 „Und fragst Du nach der Heimat, / so sagen alle, die blieben / Das Gras ist gewachsen.“ schreibt Ilse Aichinger 1955 in den dreizehn knappen Versen ihres Gedichts Breitbrunn ${ }^{21}$, einer schmerzlichen Erinnerung an die verlorene Kindheit, jener anderen Vertreibung aus der Heimat. Der hier zitierten Beschwichtigung lässt sie jedoch sofort eine Gegenüberstellung mit dem Nichtgesagten, Verschwiegenen folgen. Diejenigen, die feststellen, dass das Gras gewachsen ist, sagen „Aber nichts davon / dass die gewundenen Wege / die Hügel hinab / aufstanden und seufzten.“ (ebd.): Anamnese gegen Amnesie ... In der „Vergessenshauptstadt Wien ${ }^{22^{\prime \prime}}$ war dieses Gras allerdings nicht nur gewachsen, sondern bewusst gesät worden, und hatte das ganze, zur Idylle stilisierte und zur Touristenkulisse verkommene Land Österreich ${ }^{23}$ überwuchert. Sarkastisch-aggressiv thematisieren die Nestbeschmutzer immer wieder, dass es der beliebten alten Tiroler Volksweise ${ }^{24}$ zum Trotz gerade auf der Alm Sünde gab und gibt, und dekonstruieren in der Nachfolge von Kraus und Horvath gewissenhaft die falsche Legende.

9 Im Dienste des „anderen Österreich“ gilt es also, möglichst spektakulär das politisch korrekte Nachkriegsszenario zu kontern: Helmut Qualtinger und Carl Merz demonstrieren in ihren Produktionen satirisch Mitläufertum und Werteverfall ${ }^{25} .1969$ erscheint Gert Jonkes Geometrischer Heimatroman, weit entfernt vom Heile- Welt- 
Klischee. Turrini (Sauschlachten/1972) inszeniert bestialische Freude am Morden von Tieren und Menschen. Kein Platz für Idioten (Mitterer/1977) schildert unbarmherzige Eltern, Nachbarn, Mitmenschen. Rozznjagd (Turrini/1972) zertrümmert die Dorfidylle so wie Jelinek den Mythos Liebe und weibliche Solidarität in Die Liebhaberinnen (1975). In der Serie Alpensaga (Turrini-Pevny/1974-1979) dient die grandiose Berg- und Alpenkulisse als Hintergrund für ein immer neues Spiel der Niedertracht; die definitive Zertrümmerung des Mythos Landschaft unternimmt Elfriede Jelinek später unter anderem in Oh Wildnis, oh Schutz vor ihr (Prosa /1985) ... Alle diese Werke enthüllen, oft sehr genüsslich, eine politisch weit weniger korrekte „unheimliche Heimat ${ }^{264}$.

\section{Heimat / Museum}

10 Sogar ein Helmut Andics konnte sich am Ende der sechziger Jahre der Kritik an einer Politik, die penibel darauf bedacht war, „Museumswerte herauszuputzen ${ }^{27}$, nicht enthalten. Anknüpfung an ein verklärtes Habsburgererbe und an Brauchtum als ewige Werte wurden in einem in Abweisung (der Erinnerung, der Schuld) erstarrten Österreich in den Vordergrund gestellt und auch den Nachgeborenen, denen von der unbequemen Periode 1938-1945 möglichst wenig vermittelt wurde, als offizielle Vergangenheit vorgeführt. Nicht zufällig gehörte so zur privaten oder schulischen Pflichtlektüre der Nachkriegsgeneration neben den großen Klassikern zum Beispiel auch Peter Roseggers (eigentlich Roßegger) autobiographischer Roman Als ich noch ein Waldbauernbub war. Die 1902 erschienene erbauliche Geschichte des steirischen Buben aus einfachen bzw. ärmlichen Verhältnissen, der seine Kindheit als kleiner Hirte in Alpl bei Krieglach verbringt und später als Lehrer und Schriftsteller nicht nur den Bau der evangelischen Kirche von Mürzzuschlag, sondern auch der katholischen Kirche von Kathrein veranlasst (ganz zu schweigen von der Waldschule, die er mit Hilfe von Spenden in seinem Geburtsdorf selbst gründen kann), entspricht der nach 1945 gern gerührten ausschließlich emotionalen Saite des Heimatbegriffes. Das RoseggerDenkmal in Krieglach verdeutlicht perfekt die Legende: der junge Peter hütet verträumt seine Schafe und blickt in nicht zu beirrendem Vertrauen zum Himmel empor, der seine (im Nachhinein so vorbildlichen) Träume erfüllen wird. Heimat, das heißt hier ein konfliktfreies Dekorum duftender Almen, rauschender Tannen, fröhlich plätschernder Bäche, unschuldiger Tiere, prächtiger Blumen. Ein spärlicher aber immer zweckdienlicher und formschöner Hausrat und rauhe aber wahre Menschen, die Werte zu bewahren und zwischen Gut und Böse zu unterscheiden wissen. Besichtigen kann man diese Versatzstücke einer heilen Welt im Rosegger-Museum, das natürlich zugleich auch ein Heimatmuseum ist.

11 Um den Besuch eines Heimatmuseums inszeniert 1967 auch Gerhard Fritsch ${ }^{28}$ seinen Roman Fasching ${ }^{29}$, eine harte Abrechnung mit dem vergessensseligen und unverschämt selbstgefälligen Österreich der Nachkriegszeit. Im fünften Kapitel schildert er, wie Felix Golub, der 1945 seine Heimatstadt auf grotesk-effiziente Weise vor der Zerstörung bewahrt hatte, indem er in Frauenkleidern den Ortskommandanten zur Kapitulation zwang, der Einladung Folge leistet, als besondere Vergünstigung den neuen Schwerpunkt der Stadt noch vor seiner offiziellen Eröffnung zu besichtigen. Unter dem von seiner Braut Hilga verkündeten Motto „Sie wollen vergeben und vergessen. Auch $\mathrm{Du}$ sollst vergessen und vergeben..." (F/148) betritt Felix recht widerspenstig das Rathaus und stellt sofort fest: „Der Hitler ist weg [...]. Da ist sein Gipskopf aus Bronze 
gestanden." (F/150). Von da an verläuft der Bericht unter der doppelten Beleuchtung des (kollektiven) Vergessens und des (individuellen) Erinnerns. Über eine Wendeltreppe im düsteren Zwielicht und eine blechbeschlagene Bodentür gelangen Felix und Hilga in die zukünftigen Museumsräume selbst, wo sie unter der Führung von Fachlehrerin Radegund Plabutsch „Truhen bemalt, Truhen geschnitzt, Bauernschränke, Tische, Uhren“ (F/151) und später auch „Pferdegeschirre, Joche, kleine Madonnen“ (F/ 152) sowie „Römerfunde, Hellebarden Helme Tschakos Zunfttruhen Ladenschilder, ein[en] blecherner[n] Doppeladler, ein[en] Turmhelm“ (sic, F/155) und letzten Endes auch „tote Tauben, Mumien und Skelette in grauem Mehl“ (ebd.) erblicken. „Himmlisch“ (F/151) findet Hilga all dieses Gut. Felix jedoch beginnt - aus Ungeschicklichkeit, wie es scheint - ein Werk der Zerstörung: Der Deckel einer Brauttruhe von 1807 muss genau daran glauben wie die Krainer Krüge auf einem Wandbord. Einen energischen Verweis von Plabutsch weist er mit einem sarkastischen „Wir sind nicht im KZ“ (F/153) zurück und reagiert mit zunehmender Beklemmung auf "das stickige Gerümpel“ (F/153), das „Geratsche von den Trachten [...], dem bewahrenden Sinn des Volkes, dem natürlichen Empfinden für das ästhetisch Richtige und zweckmäßig Beste“ (F/154). Die Visite gipfelt im Saal IV in der von Direktor Wahrhejtl („unermüdlich im Aufspüren wertvollen Ahnenguts“, ebd.) naturgetreu rekonstruierten Gerichtskammer, die eine „sehenswerte Sammlung von TorturInstrumenten“ (F/157) enthält. Hier wird die Sache nun aber ernst für Felix. Man zwingt ihn fröhlich in Daumenschrauben und Beinschienen, und es wird klar, dass ihm "die Ehre“ eines Besuchs des noch nicht eröffneten Heimatmuseums nur deshalb zuteil geworden ist, weil er eine eidesstattliche Erklärung ${ }^{30}$ unterschreiben soll, mit deren Hilfe sich die „Belasteten“ Plabutsch (Bürgermeister), Plabutsch (Fachlehrerin), Wahrhejtl (Schuldirektor) und Major a.D. Lois Lubits - mit anderen Worten also die Honoratioren - einen späten „Persilschein ${ }^{31 " ~}$ verschaffen wollen.

Die Notwendigkeit einer unbekümmerten bis skrupellosen Umstilisierung des terminologisch der Nazirhetorik zuzuordnenden "Ahnengutes“ in ein neues „österreichisches Kulturerbe“ versteht sich in diesem Kontext für die Letztgenannten von selbst. Dass es sich dabei um eine in der Nachkriegszeit durchaus nicht unübliche Prozedur handelt, belegt Robert Menasse in seinem 1995 verfassten Nachwort ${ }^{32}$ durch folgende Anekdote. In einer österreichischen Kleinstadt (,in ihrer Größe etwa mit der in Fritschs Roman vergleichbar" F/241), erließ der Bürgermeister in Begeisterung des vollzogenen Anschlusses an Deutschland den Auftrag für ein Monumentalgemälde, auf dem alle Honoratioren in vollem (Nazi)Staat abgebildet waren. Nach 1945 wurde dieses Gemälde als potentielles Beweisstück für die Übereinstimmung eben dieser Honoratioren (und der Stadt überhaupt) mit der Ideologie des Dritten Reiches zum Problem. Man wollte jedoch das Geld nicht umsonst investiert haben... Und so fand man eine Lösung, „die ihre Begeisterung für dieses Bild mit den Anforderungen der geänderten politischen Lage versöhnte: Die Hakenkreuzfahnen wurden vom Künstler und zwei Gehilfen raschest mit österreichischen Farben übermalt, SS- und Wehrmachtsuniformen mutierten zu Steirer-Anzügen, aus BDM-Uniformen wurden Dirndlkleider" (F/242). Die Menschen, kommentiert Menasse, erkannten sich auch in dieser Kleidung und waren... glücklich ${ }^{33}$.

Diese glücklichen korrupten, unbarmherzigen Mitläufer und Täter von gestern, diese alten und neuen Honoratioren, die „Mörder und Irren“, wie Ingeborg Bachmann die 
ewigen Insassen des Stammtisches bezeichnet, sind allerdings, wenn es sein muss bzw. darf, durchaus erinnerungsfähig:

Sie wühlten in der Erinnerung, in manchem Dunkel, das keiner ganz preisgab, bis es dahin kam, dass ihre Gestalten sich verwandelten und wieder Uniformen trugen, bis sie dort waren, wo sie beide wieder befehligten, beide als offiziere, und Verbindung aufnahmen zum Stab; wo sie mit einer "Ju 52" hinübergeflogen wurden nach Woronesch, aber dann konnten sie sich nicht einigen über das, was sie von General Mannstein zu halten gehabt hatten im Winter 1942, und sie wurden sich einfach nicht einig, ob die 6. Armee entsetzt werden hätte können oder nicht, ob schon die Aufmarschplanung schuld gewesen war oder nicht; dann landeten sie nachträglich auf Kreta, aber in Paris hatte eine kleine Französin zu Hutter gesagt, die Österreicher seien ihr lieber als die Deutschen, und als in Norwegen der Tag heraufkam und als die Partisanen sie umzingelt hatten in Serbien, waren sie soweit - sie bestellten noch einen zweiten Liter Wein ... ${ }^{34}$

Im selben Jahr legten Carl Merz und Helmut Qualtinger ihrem Herrn Karl folgenden Kommentar in den Mund: „Alles, was man darüber spricht heute, is ja falsch ... es war eine herrliche, schöne ... ich möchte diese Erinnerung nicht missen.... ${ }^{3{ }^{4 \prime}}$.

\section{Anti-Heimatliteratur als (typisch österreichisches) Bewältigungsinstrument}

15 In diesem problematischen Österreich spalteten sich also die Lager. Im Gegenschritt zu der von einer überwältigenden Mehrheit weitgehend akzeptierten, selektiven Amnesie erklärte die Anti-Heimatkunst die offizielle österreichische Nachkriegswirklichkeit zur verkehrten Welt und ließ die in der Öffentlichkeit streng vermiedene Konfrontation mit der Vergangenheit in ihren Texten und Theaterstücken stattfinden. Sie forderte so beharrlich-demonstrativ eine umfassende Erinnerungsarbeit als Voraussetzung zu einem möglichen Verständnis der historischen Fehlentscheidungen und Fehlleistungen, die zu einer Identifikation mit der Nazi-Ideologie geführt hatten. Völlig kohärent strukturiert so Gerhard Fritsch Fasching um den oben beschriebenen Besuch im Heimatmuseum, dieser Inszenierung der Kulisse Heimat. Dessen Einrichtung wird als reine Strategie entlarvt, als eine Prozedur, durch die die Vergangenheitslüge für die Zukunft fixiert werden soll. Vor dem Kapitel die Periode 1938 - 1945, der grotesklächerliche „Bildungsroman“ des siebzehnjährigen Deserteurs Felix Golub ${ }^{36}$ und dessen (ungewollte) Heldentat. Danach ein immerwährender „Fasching“, der mit dem Zusammenbruch des Dritten Reichs beginnt: Opportunes Vergessen, Maskerade und Travestien werden zur Normalität, und ein Golub gleichzeitig zum Provokateur, dessen Bekenntnisse die bequemen Kreise empfindlich stören könnten und der daher neutralisiert werden muss: „Da war er / Noch immer gefangen / Ohne es zu wissen / Rechtskräftig verurteilt / Als größte Gefahr / Für die Bewahrung / Aller echten Werte“. Der jeweilige Beginn der sieben Kapitel des Romans, in der Inhaltsangabe so zur knappen Aussage vereint, weist in lakonischer Verkürzung auf das „Problem Golub“ hin. Nicht die Täter und Handlanger, sondern die Verweigerer und Opfer sind im neuen Österreich schuldig. Wie Felix Golub ist derjenige, der nicht vergisst, sondern Erinnerung fordert, eine Bedrohung, ein Außenseiter, dessen bloße Existenz Gefahr für das erneut Erstehende, kaum überschminkte Alte bedeutet. Der über die Fiktion formulierte derbe Ruf nach Vergangenheitsbewältigung ist als potentielle 
Infragestellung von Orientierungen, Entscheidungen, Personen eine für diese Gesellschaft unerträgliche Herausforderung.

In dem 1984 erschienenen Roman Kindernazij ${ }^{37}$ sieht sich die Hauptfigur Anatol Vitrov, wie Felix Golub ein „jugendlicher Held“, im Gegensatz zu Felix aber ein bei Kriegsende nach wie vor überzeugtes Produkt der Indoktrinierungskunst des Dritten Reiches, vor die Aufgabe gestellt, sich „umzustellen“ - das heißt hier, seinen Glauben an den nationalsozialistischen Heilsauftrag schnellstmöglich $\mathrm{zu}$ vergessen und sich den Anforderungen der neuen Lage (Sieg der Alliierten, Besetzung, Entnazifizierung und Wiederherstellung der Demokratie ...) anzupassen. „Du darfst jetzt kein Nazi mehr sein“ (K/7), erklärt ihm sein Vater und gibt ihm den Rat, die Vergangenheit als eine Filmrolle $\mathrm{zu}$ sehen, die nun beendet ist. Aber das ist trotz der unbestreitbaren Verdrängungskapazitäten der Österreicher nicht so einfach, weder für Anatol, noch für

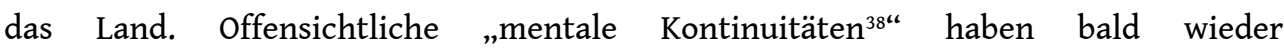
Hochkonjunktur und äußern sich in einem zunehmend banalisierten Alltagsfaschismus, den die neue Schriftstellergeneration unmittelbar thematisiert. Wie könnte es auch anders sein in einer österreichischen Nachkriegswirklichkeit, die von den restaurativen Kräften auf einem „Fundament aus Fiktionen ${ }^{39}$ aufgebaut wurde?“ (Menasse 2005, S.83). Die politisch konstruierte, zur offiziellen Realität erklärte Narrenfreiheit Österreichs wird in den Nachkriegsjahrzehnten zum offiziellen Gesellschaftsmodell, gegen das sich die Anti-Heimatliteratur demonstrativ als unerwartet effizientes Bewältigungsinstrument erweist.

Im Jahre 1703 hatte Abraham a Sancta Clara eine seiner berühmten Predigten mit folgenden Worten beschlossen: „Dergleichen verlogene Narren werden schon erfahren / wie theuer sie solche Lugen werden müssen bezahlen ${ }^{40 " ~(s i c) . ~ E b e n ~ d i e s e ~ u n b e q u e m e ~}$ Rechnung präsentiert die Anti-Heimatliteratur. In den sechziger und siebziger Jahren bricht sie polemisch das „notorische Schweigen nach der Katastrophe ${ }^{41 \text { “ }}$ und provoziert Auseinandersetzung - wenn nicht mit der Vergangenheit selbst, so zumindest mit dem von den Autoren gelieferten Skandalpotential ${ }^{42}$. Sie bringt Leben in die Kulisse, indem sie die unbequemen Themen in den Vordergrund rückt und die Frage nach der Verantwortung stellt. Das „offizielle“ Österreich lehnt die Diskussion ab? Die AntiHeimatliteratur zwingt sie dazu, ihre „bösen“ Werke zumindest in der Form der üblen Nachrede wahrzunehmen. Sie dekonstruiert systematisch die falsche Versöhnungspolitik der Nachkriegszeit und Wiederaufbauepoche, und enthüllt das Fragwürdige einer Gesellschaft, die Erinnerung als systemstörend zurückweist und es vorzieht, sich allein durch die materiellen Erfolge des Wirtschaftswunders zu legitimieren. Diese Gesellschaft hat das 1946 von Ilse Aichinger geforderte Misstrauen gegen sich selbst vergessen - jenen Selbstschutz gegen die sentimentale Beschönigung allzu menschlichen Handelns, der zu gegebener Zeit vielleicht hätte die Kraft bringen können, sich dem allzu Unmenschlichen zu verweigern. Die österreichische AntiHeimatliteratur ist somit als ein essentieller Beitrag zum Thema Geschichtsvermittlung und Geschichtsverständnis $\mathrm{zu}$ verstehen, als ein unumgängliches Instrument der Vergangenheits- bzw. Gegenwartsbewältigung - eine Funktion, die sie auch heute noch höchst effizient zu erfüllen versteht. 


\section{NOTES}

1. Gesamtschüler Ulrich, 13. In : Was ich über Adolf Hitler gehört habe ... Folgen eines Tabus: SchülerAufsätze von heute, herausgegeben von Dieter Bossmann, Frankfurt/Main, Fischer-Verlag, 1977, S. 316.

2. Das Volkstheater bei Horvath, der Trivialroman bei Jelinek usw.

3. Und manchmal auch sehr konkret, zum Beispiel durch das Abladen von Mistfuhren vor den Aufführungsstätten unerwünschter Theaterstücke (Heldenplatz, Burgtheater ...).

4. Vgl. Robert Menasse, Das war Österreich, Frankfurt/Main, Suhrkamp, 2005, S.101.

5. Vgl. Christine Lecerf, Jacques Lajarrige, Yves Masson in ihrem Vorwort zu dem Band „Littérature d'Autriche“, Europe, n866-867, 2001. S.3-7.

6. A.a.O.

7. In Anlehnung an Uwe Johnson, Jahrestage IV [1983], Frankfurt/Main, edition suhrkamp, 1993. Marie zu ihrer Mutter Gesine Cresspahl: „Endlich sind wir angekommen, wo meine Erinnerung Bescheid weiß.“(S.1875).

8. Heimat als Utopie, Frankfurt/Main, Suhrkamp, 2000, S.32.

9. Dazu unter anderen Andrea Kunne: Heimat im Roman: Last oder Lust, Transformationen eines Genres in der österreichischen Nachkriegsliteratur, Amsterdam-Atlanta, Rodopi, 1991.

10. Zu diesem Thema siehe Breuss, Liebhart, Pribersky: Inszenierungen. Stichwörter zu Österreich. Mit einem Vorwort von Anton Pelinka. Wien, Sonderzahl, 1995, S.320-330.

11. Wien, Juli 1945. Das Bildmaterial für die Darstellung dieser heilen, jedoch durchaus menschenleeren Welt wurde dabei übrigens großteils aus Bruno Brehms 1938 erschienenem Band Glückliches Österreich übernommen (siehe Menasse, a.a.0., S.98/99).

12. Für ihn ohne große Differenzierung Kaiserzeit, Heuriger, Anschluss ...

13. Carl Merz/Helmut Qualtinger: Der Herr Karl [1961], in: Traugott Krischke Hrsg., Helmut Qualtinger. Gesammelte Werke, Band 1 („Der Herr Karl“ und andere Texte fürs Theater), Wien, Deuticke, 1995, S.183.

14. Ich übernehme diesen Begriff von Gertraud Steiner, Die Heimatmacher. Kino in Österreich 1946-1966, Wien, Verlag für Gesellschaftskritik, 1987. Die ausschlaggebende Rolle des neuen österreichischen Heimatfilms nach 1945 in der strategischen Wiederherstellung von Macht- und Gesellschaftsstrukturen muss ganz besonders unterstrichen werden.

15. Bzw. nicht zurückgerufen wurden, wie z.B. die ausgewanderten Juden.

16. Auf das Problem des kulturellen Auseinanderfallens des Landes wies u.a. Otto Schulmeister hin und stellte die Frage, „in welchem Österreich die neue Republik letzten Endes stattfinden sollte": Im Land des Herrn Karl (Merz/Qualtinger) und der Alpensaga (Turrini) oder im Land des Mayerling-Lipizzaner-Sängerknaben-Klischees ... In: Der zweite Anschluss. Österreichs Verwandlung seit 1945, Wien-München-Zürich-Innsbruck, Fritz Molden-Verlag, 1979, S.118.

17. Vgl. Menasse, a.a.O., S.101.

18. Die Publikumsreaktionen zum Monolog Der Herr Karl kommentierte der Kritiker Hans Weigel in der Kronenzeitung vom 2.12.1961 wie folgt: „, Der Herr Karl wollte einem Typus auf die Zehen treten, und ein ganzes Volk schreit ,Au'!“. Zitiert in Helmut Qualtinger. Gesammelte Werke, Band 1, op. cit., S.361.

19. Dieser Satz beschließt Inge Merkels Roman Das andere Gesicht [1982], Frankfurt/Main, Fischer, 1995, S.292.

20. Frei nach Elfriede Gerstel zitiert in Inszenierungen, a.a.O., S. 142.

21. In : Richard Reichensperger Hrsg., Ilse Aichinger. Gesammelte Werke. Bd. 8 („Verschenkter Rat“), Frankfurt/Main, Fischer-Verlag, 1991, S.18. 
22. Vgl. Robert Schindels Gedicht „Vineta 1“, in: Ein Feuerchen im Hintennach. Gedichte von 1986-1991, Frankfurt/Main, edition suhrkamp, 1992.

23. Wendelin Schmidt-Dengler konnte so die Anti-Heimatliteratur als die Zerschlagung der Idylle definieren (vgl. „Die antagonistische Natur. Zum Konzept der Anti-Idylle in der neueren österreichischen Prosa“, in: Literatur und Kritik, Nr.40, 1969, S.577-585).

24. „Auf der Alm, da gibt's koa Sünd!“. Leitfaden der in Österreich lange so beliebten Löwingerbühne. Der Titel wurde auch für einen der erfolgreichsten österreichischen Heimatfilme verwendet (Franz Antel/Franz Josef Gotlieb 1950, mit Annie Rosar, Susi Nicoletti, Sissy Löwinger (!), Rudolf Carl ...), in den siebziger Jahren für eine Serie von Sexklamaukfilmen. Auch heute ist er übrigens noch aktuell, zum Beispiel als Avatar in Second Life ...

25. Der Herr Karl (vgl. Fußnote 12), und, um nur ein Beispiel zu geben, der Sketch „Die Ahndlvertilgung" [1956], in dem Kinder und Kindeskinder das Ableben (und somit den Erbprozess) ehrwürdiger Altbauern skrupellos beschleunigen. In: Qualtinger's beste Satiren. Vom Travnicek zum Herrn Karl, Wien, Langen-Müller, 1973, S. 16-18.

26. Vgl. der Titel der Essays zur österreichischen Literatur von W.G.Sebald, Frankfurt/Main, Fischer, 1995.

27. In: Die Insel der Seligen. Österreich von der Moskauer Deklaration bis zur Gegenwart, WienMünchen-Zürich, Molden MTV, 1968, S.221.

28. Geboren 1924 in Wien, Selbstmord 1969.

29. Erstveröffentlichung bei Rowohlt. Neu aufgelegt bei Suhrkamp, Frankfurt/Main, 1995. In der Folge Kürzel F. Alle Zitate sind dieser zweiten Ausgabe entnommen.

30. „In Zusammenwirkung mit Frau Baronin Pisani haben die Genannten im Jahr 1945 ungeachtet der damit verbundenen Gefahren und trotz Missbilligung meiner Fahnenflucht mir aus humanitären Gründen jede Unterstützung angedeihen lassen und mich meinem Wunsch gemäss als weibliche Person behandelt. Alle Gerüchte bezüglich einer anderen Handlungsweise der Obgenannten, wann und wo sie verbreitet worden sind, entbehren ebenso jeder Grundlage [....]“ (F/159-160). Von mir hervorgehoben.

31. Unschuldsbeweis nach 45 .

32. S.239- 249 .

33. Fritsch scheint diese Anekdote nicht gekannt zu haben.

34. „Unter Mördern und Irren“, in: Das dreißigste Jahr. Erzählungen, München, Piper, 1961, S.68.

35. Ebd., S.174/175. Von mir hervorgehoben.

36. „Grenadier, geboren am 3.6.1927 in Graz, fahnenflüchtig seit 31.12.1944 ....“ (F/60).

37. Von Andreas Okopenko. Wiederaufgelegt 1999 im Ritter-Verlag (Klagenfurt-Wien). Kürzel K.

38. Robert Menasse: Das war Österreich. Gesammelte Essays zum Land ohne Eigenschaften, Frankfurt/ Main, Suhrkamp, 2005, S.102.

39. Als zur Anti-Heimat mutierende Märchen bezeichnete diese Fiktionen Prof. Dr. Johann Sonnleitner (Universität Wien) anlässlich des Ilse Aichinger - Kolloquiums in Rouen (18./19. Mai 2007).

40. „Ein Verlogner Narr“, in: Alois Haas Hrsg., Abraham a Sancta Clara: Wunderlicher Traum vom grossen Narrennest, Stuttgart, Reclam (Universalbibliothek) Nr.6399, 1969, S.30.

41. Vgl. Robert Schindel: „Schweigend ins Gespräch vertieft. Anmerkungen zu Geschichte und Gegenwart des jüdisch-nichtjüdischen Verhältnisses in den Täterländern“, in: Mein Liebster Feind. Essays, Reden, Miniaturen, Frankfurt/Main, Suhrkamp, 2004, S.19.

42. Als Beispiel sei hier nur die Rezeption von Fritschs Roman Fasching erwähnt, der auf heftigste Ablehnung stieß, jahrelang vergriffen war und erst 1995 wieder aufgelegt wurde. 


\section{RÉSUMÉS}

Gesellschaftskritik hat in Österreich Tradition und Methode. Seit Nestroy (über Kraus, Horvath und später Turrini, Bernhard, Jelinek usw.) enthüllen die sogenannten „Nestbeschmutzer“ die Fehlleistungen, Brüche und Lügen des Systems, und übernehmen die Funktion der Gegenspieler der Institutionen, die ebenso wie die breite Öffentlichkeit (bzw. das Publikum) mit Verstörung und Aggressivität auf die Herausforderung dieser unbequemen Produktionen reagieren.

Nach 1955 spitzt sich der Konflikt zwischen dem offiziellen, auf den von den Alliierten gewährten höchst gelegenen neuen Gründungsmythen beruhenden und wieder souveränen Österreich und der Kritik der Nestbeschmutzer zu. Er kristallisiert sich in den sechziger und siebziger Jahren um die als fragwürdig empfundene Inszenierung des Begriffs „Heimat“, und führt so zur Herausbildung einer „spezifisch österreichischen“ Gattung, der Anti-Heimatliteratur, die wir hier als ein unumgängliches Instrument der Vergangenheitsbewältigung verstehen und analysieren.

L'Autriche peut se prévaloir de la longue tradition d'une littérature (et d'un théâtre!) qui interroge - le plus souvent par une critique de la langue par la langue - les dysfonctionnements cachés de la société et du système. Se focalisant plus particulièrement sur les ratés de l'Histoire, les fameux «Nestbeschmutzer» (de Nestroy, Kraus, Horvath à, plus tard, Turrini, Bernhard, Jelinek et bien d'autres) provoquent et heurtent les institutions, et un public peu enclin à accepter une démarche refusant toute forme d'apologie non distanciée des instances dirigeantes. Après 1955, le conflit entre la définition officielle de la nouvelle Autriche souveraine et la remise en question de ses nouveaux et fort accommodants mythes fondateurs s'articule essentiellement autour du concept de «Heimat». Dans les années soixante / soixante-dix naît ainsi un genre nouveau qui est sans doute spécifique à l'Autriche, la «Anti-Heimatliteratur» que nous étudierons ici en tant que lieu de confrontation du pays avec son Histoire et par conséquent en tant qu'instrument indispensable d'appropriation du passé.

\section{INDEX}

Mots-clés : Anti-Heimatliteratur

\section{AUTEUR}

\section{INGEBORG RABENSTEIN-MICHEL}

Université Lyon I - Claude Bernard/IUFM 